\title{
NOTIFICACIÓN DE ACCIDENTES Y ENFERMEDADES LABORALES AL MINISTERIO DE TRABAJO. PERÚ 2010-2014
}

\author{
Christian R. Mejia ${ }^{1,2, a}$, Matlin M. Cárdenas ${ }^{3, b}$, Raúl Gomero-Cuadra ${ }^{4, c}$
}

\begin{abstract}
RESUMEN
Con el objetivo de determinar las tendencias de los accidentes y enfermedades laborales notificadas al Ministerio de Trabajo del Perú, se realizó un estudio descriptivo de datos secundarios, con los reportes extraídos de los boletines mensuales desde septiembre de 2010 a diciembre de 2014. Se notificaron a nivel nacional 54596 accidentes laborales no mortales, las tasas de los accidentes laborales no mortales por millón de habitantes aumentaron en el período 20112013 (296,5 el 2011, 955,9 el 2012 y 1176,3 el 2013), disminuyendo en el 2014 (878,9). Hubieron 674 accidentes mortales, cuyas tasas se incrementaron entre el 2011-2012 y se redujeron entre el 2013-2014. Se reportaron 346 enfermedades laborales, las más frecuentes fueron los casos de hipoacusia (77), enfermedades por posturas inadecuadas (57) y dermatitis alérgica (44); los reportes se redujeron de 6,9 en el 2011 a 2,3 en el 2014. Los reportes de enfermedades laborales fueron disminuyendo, lo que podría indicar una subnotificación importante.
\end{abstract}

Palabras clave: Notificación de accidentes del trabajo; Salud laboral; Enfermedades laborales (fuente: DeCS BIREME)

\section{NOTIFICATION OF ACCIDENTS AND OCCUPATIONAL DISEASES TO THE MINISTRY OF LABOR. PERU 2010-2014}

\begin{abstract}
In order to determine trends in accidents and illnesses reported to the Ministry of Labor of Peru, a descriptive study of secondary data from the reports of monthly newsletters from September 2010 to December 2014 was performed. At the national level, 54,596 non-fatal accidents were reported. The rates of non-fatal accidents increased in 2011-2013 (296.5 in 2011, 955.9 in 2012 and 1176.3 in 2013), decreasing in 2014 (878.9). There were 674 fatal accidents whose rates increased between 2011 and 2012 and were reduced between 2013 and 2014. 346 occupational diseases were reported; the most frequent were cases of hearing loss (77), illness by inadequate postures (57) and allergic dermatitis (44). Reports declined from 6.9 in 2011 to 2.3 in 2014. Reports of occupational diseases were declining, which could indicate significant underreporting.
\end{abstract}

Key words: Occupational accidents registry; Occupational health; Occupational diseases (source: MeSH NLM).

\section{INTRODUCCIÓN}

Los accidentes laborales están definidos como todo suceso repentino que sobrevenga por causa o con ocasión del trabajo, y que produzca en el empleado una lesión orgánica, una perturbación funcional, una invalidez o la muerte. Las enfermedades laborales son aquellas contraídas por la exposición a factores de riesgo que resulten de la actividad laboral. A nivel mundial se calcula que, aproximadamente, dos millones de personas mueren anualmente por causa de accidentes laborales, en tanto que las enfermedades profesionales siguen siendo una de las principales causas de muertes relacionadas con el trabajo ${ }^{(1)}$. Esto se asocia con múltiples factores, como las condiciones de seguridad del lugar de trabajo, el puesto laboral en el que se desempeñan o el ámbito en el cual laboran ${ }^{(2,3)}$; lo que genera repercusiones en el trabajador, su familia y la sociedad (4).

La notificación oportuna de accidentes y enfermedades laborales conlleva a que se detecten condiciones inseguras a las que estarían expuestos los trabajadores. Además, a partir de ello se pueden establecer nuevas políticas y estrategias para la mejora en la seguridad del

\footnotetext{
Escuela de Medicina Humana, Universidad Continental. Huancayo, Perú.

Asociación Médica de Investigación y Servicios en Salud. Lima, Perú.

Facultad de Medicina, Universidad Ricardo Palma. Lima, Perú.

Universidad Peruana Cayetano Heredia. Lima, Perú.

Médico magíster en Salud Ocupacional; ${ }^{\mathrm{b}}$ estudiante de Medicina; ${ }^{\mathrm{c}}$ médico especialista en Medicina Ocupacional y Medioambiente.

Recibido: : 01-09-14 Aprobado: 18-03-15
} 
trabajador ${ }^{(5)}$. Si bien las instituciones estatales tienen el deber de publicar las cifras de estos acontecimientos -siendo el Ministerio del Trabajo (MINTRA) el encargado en nuestro medio-, son pocos artículos de investigación que reportan el agregado de varios meses o un período temporal amplio ${ }^{(6)}$; por lo que resulta importante la evaluación de las tendencias para el seguimiento de las estrategias previamente establecidas ${ }^{(7)}$.

Existen algunos reportes de tasas, de sectores específicos de la industria, relacionados con la accidentabilidad y morbilidad en el trabajo. Sin embargo, no se encontró publicaciones que abarquen a todo el sector productivo nacional ${ }^{(8)}$. Por tal motivo, el objetivo del presente trabajo fue determinar la tendencia en las notificaciones de accidentes y enfermedades laborales según los reportes del MINTRA, entre el 2010-2014.

\section{EL ESTUDIO}

Estudio retrospectivo, descriptivo, de análisis de datos secundarios. Se analizaron las notificaciones de accidentes y enfermedades laborales realizadas a nivel nacional, obtenidos de los boletines mensuales publicados en la página web del MINTRA (www.mintra. gob.pe), desde septiembre de 2010 hasta diciembre de $2014{ }^{(9)}$. Se incluyeron todos los datos reportados y completos en este período (tasa de pérdida: 0\%). La muestra fue no aleatoria de tipo censal.

Se obtuvo los datos de las notificaciones de los accidentes laborales no mortales, según el total y los lugares con mayor cantidad de notificaciones (Lima metropolitana, Callao, Piura, Arequipa y La Libertad). Asimismo, los datos de las notificaciones de los accidentes mortales, las enfermedades (se extrajo la información según cada una de las enfermedades notificadas) y los incidentes laborales (definido como el suceso inesperado relacionado con el trabajo que puede o no resultar con daños a la salud. En un sentido más amplio, incidente involucra todo tipo de accidente de trabajo) ${ }^{(10)}$.

Estos datos fueron recolectados por el MINTRA, a través de las instituciones encargadas y el sistema de reporte que manejan a nivel nacional. En el caso de los accidentes e incidentes, una vez acontecido el suceso, los trabajadores que acuden a un establecimiento de salud (EsSalud o EPS, según su tipo de aseguramiento) brindan la información de cómo sucedió el hecho a través de una ficha de recolección. En el caso de las enfermedades laborales, estas se confirman por medio de una investigación judicial (que tiene como fin, determinar si la enfermedad fue producida a causa del trabajo) o por encargo a una comisión que se genera para investigar cada caso (por encargo de alguna entidad prestadora de salud); siendo reportada una vez que se confirme que se trata de una enfermedad laboral. Las fichas de reporte para cada uno de estos acontecimientos se encuentran en la página oficial del MINTRA ${ }^{(9)}$.

Se procedió a la descarga de cuarenta boletines de reportes; esta información fue consignada en una base de datos, posteriormente, se realizó el control de calidad de los datos, los cuales se verificaron uno a uno, hasta en tres oportunidades.

Para el análisis de los datos, las variables categóricas fueron representadas por frecuencias y porcentajes. Para el análisis de la tendencia temporal se usó el generador de gráficos del programa Microsoft Excel; se incluyó la ecuación y el coeficiente de la línea de tendencia, así como los valores inicial, final y los valores extremos.

Los datos son de dominio público, se contó con la aprobación del Comité de Ética del Hospital San Bartolomé (1375-DG-OADI-N. ${ }^{\circ} 0383$-HONADOMANI-SB-2014).

\section{RESULTADOS}

En el período de estudio se encontró 54596 reportes de accidentes laborales no mortales, de los cuales el $90,2 \%$ (48 365) fueron en varones. Lima Metropolitana fue la ciudad en donde se reportó la mayor cantidad de accidentes laborales no mortales $(76,9 \%)$, seguida de la provincia constitucional del Callao $(15,0 \%)$ y el departamento de Arequipa (3,8\%). En el mismo período se reportaron 674 accidentes mortales, 3432 incidentes y 346 enfermedades laborales. Las cifras muestran en la Tabla 1.

Tabla 1. Accidentes laborales no mortales, mortales, incidentes y enfermedades notificadas al Ministerio del Trabajo de Perú 2010-2014

\begin{tabular}{|c|c|c|c|c|c|c|}
\hline Notificación de & $2010^{*}$ & 2011 & 2012 & 2013 & 2014 & Total \\
\hline $\begin{array}{l}\text { Accidentes } \\
\text { laborales no } \\
\text { mortales }\end{array}$ & 198 & 4728 & 15508 & 19412 & 14750 & 54596 \\
\hline $\begin{array}{l}\text { Lima } \\
\text { Metropolitana }\end{array}$ & 140 & 4117 & 11630 & 14804 & 11271 & 41962 \\
\hline Callao & 0 & 301 & 3430 & 3481 & 991 & 8203 \\
\hline Arequipa & 0 & 29 & 183 & 222 & 1647 & 2081 \\
\hline Piura & 14 & 100 & 410 & 531 & 413 & 1468 \\
\hline La Libertad & 0 & 20 & 79 & 101 & 87 & 287 \\
\hline $\begin{array}{l}\text { Accidentes } \\
\text { laborales } \\
\text { mortales }\end{array}$ & 24 & 145 & 199 & 178 & 128 & 674 \\
\hline $\begin{array}{l}\text { Incidentes } \\
\text { laborales }\end{array}$ & 130 & 623 & 826 & 983 & 870 & 3432 \\
\hline $\begin{array}{l}\text { Enfermedades } \\
\text { laborales }\end{array}$ & 8 & 110 & 107 & 82 & 39 & 346 \\
\hline
\end{tabular}

*Meses septiembre-diciembre 


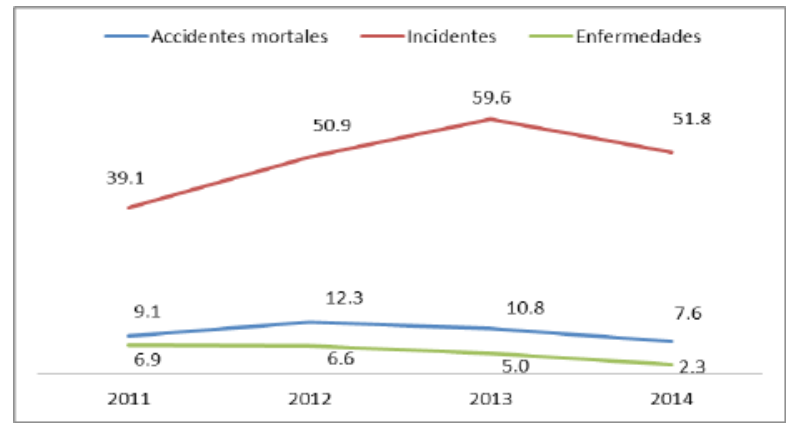

Figura 1. Tasas de reporte de accidentes laborales mortales, incidentes y enfermedades laborales en el Perú, calculadas como número de eventos sobre el número de trabajadores de población económicamente activa (PEA) peruana por millón de habitantes

Al obtener las tasas de las notificaciones por millón de personas que pertenecían a la población económicamente activa (PEA), se observó que las tasas de los accidentes laborales no mortales aumentaron en el período 2011-2013 (296,5 el 2011; 955,9 el 2012, y 1176,3 el 2013), disminuyendo en el 2014 (878,9); esta tendencia fue similar para las tasas de reporte de los incidentes laborales. Los accidentes laborales mortales tuvieron un incremento en sus tasas de reportes entre el 2011-2012, pero en el período del 2013-2014 se redujeron. En cambio, las tasas de reportes de enfermedades laborales siempre se redujeron, siendo en el último año menos de la mitad que el anterior reporte anual. Las tasas de reportes se muestran en la Figura 1.

La Figura 2A muestra la tendencia de las notificaciones mensuales de accidentes laborales no mortales, en total y en las dos ciudades con mayor cantidad de reportes (Lima Metropolitana y la provincia constitucional del Callao); se observa una tendencia al incremento de las notificaciones en estas ciudades. La Figura 2B muestra la tendencia de los reportes de accidentes laborales mortales, se observa una leve tendencia al aumento de las notificaciones; siendo los picos máximos cifras entre 25-29 muertes por mes; cabe resaltar que desde mayo de 2013 no se superan los 20 muertos por reporte mensual. La Figura $2 \mathrm{C}$ muestra una tendencia de cada vez menos reportes de enfermedades laborales, sin superar la docena de enfermedades desde hace dieciocho meses.

Las enfermedades laborales más reportadas en los 52 meses de publicaciones del MINTRA fueron: la hipoacusia (77 reportes), seguida de las causadas por posturas inadecuadas en el trabajo (57), dermatitis alérgica (44), lumbago (37) y la silicosis (35) (Tabla 2).
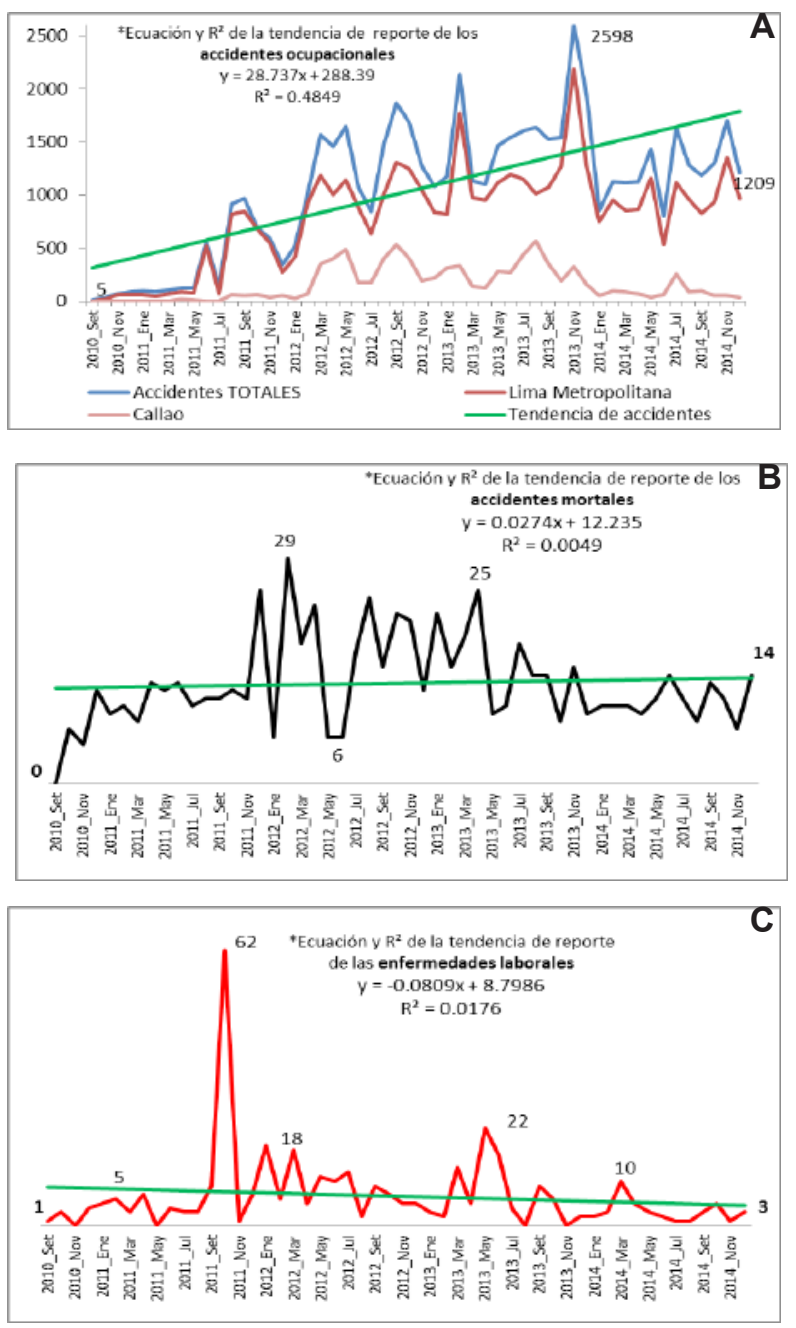

Figura 2. A: Tendencia de los accidentes laborales no mortales. $B$ : accidentes laborales mortales y C: enfermedades laborales, notificadas al MINTRA, 2010-2014

Tabla 2. Enfermedades laborales notificadas al Ministerio de Trabajo, 2010-2014

\begin{tabular}{lcccccc}
\hline \multicolumn{1}{c}{ Enfermedad } & $\mathbf{2 0 1 0}^{*}$ & $\mathbf{2 0 1 1}$ & $\mathbf{2 0 1 2}$ & $\mathbf{2 0 1 3}$ & $\mathbf{2 0 1 4}$ & Total \\
\hline Hipoacusia & 0 & 28 & 21 & 24 & 4 & 77 \\
Dermatitis alérgica & 0 & 3 & 30 & 7 & 4 & 44 \\
Lumbago & 0 & 9 & 6 & 18 & 4 & 37 \\
Silicosis & 0 & 4 & 17 & 5 & 9 & 35 \\
Por posturas & 0 & 38 & 4 & 15 & 0 & 57 \\
inadecuadas & 0 & 7 & 5 & 2 & 3 & 17 \\
Leishmaniosis & 0 & 9 & 5 & 0 & 2 & 16 \\
A causa de tóxicos/ & 0 & 0 & 3 & 0 & 0 & 3 \\
químicos & 0 & 0 & 0 & 0 & 1 & 1 \\
Dorsalgia & 0 & 0 & 0 & 1 & 0 & 1 \\
Infecciones víricas & 0 & 0 & 1 & 0 & 0 & 1 \\
Varices en miembros & 0 & 1 & 1 & 0 & 0 & 2 \\
inferiores & 0 & 0 & 1 & 0 & 0 & 1 \\
Ciática & 0 & 15 & 0 & 0 & 4 & 19 \\
A causa de radiaciones & 0 & & \\
Hepatitis & Otras causas & & & & &
\end{tabular}

${ }^{*}$ Meses septiembre-diciembre 


\section{DISCUSIÓN}

Esta investigación sobre las tendencias de los accidentes y enfermedades laborales resulta importante por ser un análisis situacional que puede generar recomendaciones (hacia los empleadores, el gobierno y otras entidades relacionadas) a fin de generar cambios para la mejora de dichos indicadores. Esto, debido a que se requiere que la fuerza laboral se encuentre en plena capacidad de trabajar, para poder generar un desarrollo sostenido en el tiempo (la PEA peruana en el período de estudio superó los 15 millones) ${ }^{(9)}$. Puesto que, un accidente o una enfermedad acontecida, no solo trae repercusiones para la empresa o para la integridad del propio trabajador, sino que también afecta económicamente a la familia, a la sociedad y al estado ${ }^{(4,11)}$.

En más de cuatro años de notificaciones, la mayoría de reportes ocurrieron en la capital peruana, y el resto se dividió en cuatro provincias. Esto puede deberse al centralismo que genera la concentración de la PEA en estos cinco lugares; la escasa cantidad de reportes en las otras diecinueve provincias puede deberse a múltiples causas, siendo la principal el hecho de tener una menor cantidad de empresas ${ }^{(12)}$. Otra causa puede ser una subnotificación, que algunas investigaciones demuestran que se da en el ámbito laboral entre el 26 a $78 \%{ }^{(5,13,14)}$. Esto atenta contra la salud de los trabajadores y genera vacíos en las estadísticas nacionales.

Según las gráficas de tendencia, el aumento de las notificaciones de los accidentes laborales no mortales en los primeros años se puede deber a múltiples factores, como la mayor cantidad de empresas (que se crearon a partir del 2009), sobre todo en la ciudad capital y en los rubros de extracción de materias primas ${ }^{(15)}$. También puede deberse a la promulgación de la Ley 29783 en agosto de 2011, la cual reglamenta la notificación que deben realizar las empresas cuando alguno de sus trabajadores sufre un accidente ${ }^{(16)}$.

Si bien en los primeros años hubo un leve incremento en las notificaciones de los accidentes mortales, estos disminuyeron en los últimos dos años, ello puede deberse al aumento de la tecnología industrial, de manera que muchos procedimientos riesgosos para el trabajador han disminuido notablemente ${ }^{(17)}$.

Además, las empresas y el Estado están implementando estrategias para aminorar el riesgo, impartiendo capacitaciones a los trabajadores, brindándole material de seguridad, realizando inspecciones por parte de la Superintendencia Nacional de Fiscalización Laboral (SUNAFIL), entre los más destacables ${ }^{(18)}$. Aún no se ha llegado a generar una completa equidad de la salud y seguridad en el trabajo ${ }^{(19)}$, pero este es un proceso que es paulatino, sobre todo dependiente de los actores principales (el estado, las empresas y los trabajadores), ya que incluso algunos países desarrollados, y con tecnología de punta, todavía reportan fluctuaciones en sus reportes seculares de tasas de accidentes mortales y no mortales ${ }^{(20)}$. Por lo que se deben seguir generando evidencias que contribuyan a la mejora del statu quo en este rubro.

Por último, en el reporte de enfermedades laborales se halló una disminución en la tendencia mensual y en sus tasas anuales, con una cantidad de reportes menor a la esperado, incluso para enfermedades laborales tan comunes como la hipoacusia, las enfermedades generadas por posturas inadecuadas y las de origen psicosocial; lo que contrasta con diversas investigaciones nacionales e internacionales, que muestran un número bastante superior a lo reportado a nivel nacional en los

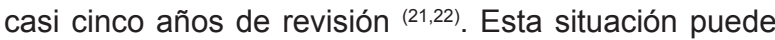
deberse a una subnotificación, que parte desde la base de no tener adecuadas definiciones o por un inadecuado uso de la lista de enfermedades profesionales.

Se observó que el MINTRA notifica como enfermedades algunos síntomas o signos patológicos específicos; así como la superposición de enfermedades específicas y otras que engloban a un grupo de ellas. Se sugiere que para evitar esto, se deben generar normas claras y concisas, basadas en la realidad nacional, como los realizados por algunos países vecinos ${ }^{(23,24)}$.

Otras causas para esta disminución, pueden ser: la falta de notificación por aquellos que están obligados a reportarlas (que son los establecimientos de salud que detectan la patología, según la Resolución Ministerial 480-2008) (5); la deficiente cantidad de médicos idóneos para realizar dichos diagnósticos (25); el temor del trabajador de reportar la enfermedad por las sanciones; entre otros. Esta subestimación de la real situación debe ser evaluada y corregida, de lo contrario en algunos años se generarán un aumento en los años de vida potencialmente perdidos (AVPP) y costos elevados por la atención de salud de estos trabajadores ${ }^{(26)}$, sobre todo cuando pasen a formar parte de la población adulto mayor.

Como limitación se puede mencionar el sesgo de información, puesto que los datos fueron obtenidos por el ente rector de nuestro país, pudiéndose haber generado una subnotificación en los reportes, ocasionando que las cifras mostradas no reflejen la actual realidad nacional; a pesar de esto creemos que los resultados mostrados son importantes, porque informan la única realidad sobre este problema, que conocemos a nivel nacional, hasta el momento. Otra limitación fue que la clasificación de algunas enfermedades profesionales no 
seguía estándares internacionales, fueron basadas en el listado de enfermedades profesionales del MINSA, y en algunos casos no son enfermedades (lumbago o dorsalgia) o también unos pueden incluir a otros (hepatitis y enfermedad vírica). Por último, las tasas no se pudieron calcular según horas hombre, debido a que no se contaba con estas cifras; lo que hubiese sido útil para calcular el impacto según la exposición real en los trabajadores, aun así estas tasas pueden servir para hacer comparaciones con otras realidades.

Se concluye que la tendencia de las notificaciones de accidentes laborales no mortales se ha incrementado desde hace algunos años. Los accidentes laborales mortales han aumentado ligeramente en su notificación mensual. Además, las enfermedades laborales son reportadas en una mínima frecuencia y con tendencia a la disminución. Se recomienda generar métodos de vigilancia de las notificaciones, ya que no basta con promulgar una ley, sino además, velar por su cumplimiento y constatar que se realicen los reportes.

Contribuciones de autoría: CRM y MMC tuvieron la idea de investigación, diseñaron el estudio y recopilaron los datos, CRM y RGC realizaron el análisis e interpretación de datos, todos los autores participaron en la redacción del manuscrito y aprobaron la versión final.

Fuentes de financiamiento: autofinanciado.

Conflictos de interés: los autores declaran no tener conflictos de interés.

\section{REFERENCIAS BIBLIOGRÁFICAS}

1. Organización Internacional del Trabajo, Departamento de Comunicación. Dos millones de muertes por accidentes laborales cada año [Internet]. Ginebra: Organización Internacional del Trabajo; 2002 [citado el 10 de marzo de 2015]. Disponible en: http://www.ilo.org/global/aboutthe-ilo/media-centre/press-releases/ WCMS_071435/lang--es/index.htm

2. Salanova Soria M, Martínez Martínez I, Lorento Prieto L. La relación entre el exceso de confianza y los accidentes laborales en trabajadores de la construcción: un estudio cualitativo. Gestión Práctica de Riesgos Laborales. 2011;(86):8-13.

3. García AM, Gadea R, Sevilla MJ, Ronda E. Validación de un cuestionario para identificar daños y exposición a riesgos ergonómicos en el trabajo. Rev Esp Salud Pública. 2011;85(4):339-49.

4. Rubio MIM. Estudio de costes de los accidentes laborales. España 20072011 [Internet]. Barcelona: Mutua Universal; 2012 [citado el 10 de marzo de 2015]. Disponible en: http://www. amat.es/Ficheros/14507.pdf

5. Haro-García LC, Juárez-Pérez CA, Sánchez-Román FR, Aguilar-Madrid G. Panorama del subregistro de los accidentes y enfermedades de trabajo en México. Rev Médica Risaralda. 2014;20(1):47-9.

6. Chile, Ministerio de Salud. Informe perfil epidemiológico de los accidentes de trabajo con resultados de muerte en la región metropolitana años 2003 2007 [Internet]. Santiago: SEREMI de Salud; 2008 [citado el 10 de marzo de 2015]. Disponible en: http:// www.asrm.cl/archivoContenidos/ ACCIDENTES_TRABAJO FATALES_2003_2007.pdf

7. Benavides FG, López-Ruiz M, Castejón E. Tendencia secular de las lesiones por accidentes de trabajo en España, 1904-2005. Arch Prev Riesgos Labor. 2008;11(3):141-7.

8. Carrasco Villadoma C. Comportamiento epidemiológico de la atención médica en los trabajadores fabriles de una compañía cervecera, en la ciudad de Lima, período: años 2004 a 2006. Acta Med Per. 2010;27(2):105-18.

9. Trabajo.gob.pe [Internet]. Lima: Ministerio de Trabajo y Promoción del Empleo; 2015 [citado el 10 de marzo de 2015]. Disponible en: http://www. trabajo.gob.pe/mostrarContenido. php id $=730 \&$ tip $=86$

10. Perú, Ministerio de Energía y Minas. Decreto supremo que aprueba el reglamento de seguridad y salud ocupacional y otras medidas complementarias en minería [Internet]. Lima: MINEM; 2010 [citado el 10 de marzo de 2015]. Disponible en: http://www. minem.gob.pe/_legislacion.php?idSector $=18$ idLegislacion $=6013$ ? ixlwjsbm fbclwjec

11. Ruiz F, Palomino JC, Gomero R, Llap C. Prevalencia, impacto en la productividad y costos totales de las principales enfermedades en el personal hospitalario en un hospital al sur del Perú en el año 2003 [Internet]. Moquegua: 2003 [citado el 10 de marzo de 2015]. Disponible en: http:// www.bvsde.paho.org/bvsacd/cd27/ prevalencia.pdf

12. Vargas Morales ZE. Centralismo, descentralizacion y expectivas de la ciudadania (Conchucos BajoRegion Ancash). Investig Sociales. 2014;12(21):203-20.

13. Agelvis J. Caracterización de trabajadores que sufrieron accidentes laborales con afectación de miembros superiores, atendidos por el Instituto Nacional de Prevención, Salud y Seguridad Laborales (INPSASEL) Sede Aragua, año 2010 [Internet]. Aragua: INPSASEL; 2012 [citado el 10 de marzo de 2015]. Disponible en: http://mriuc.bc.uc.edu. ve/handle/123456789/117

14. Alarcón Peralta C. Análisis de los factores que inciden en la presencia de accidentes laborales con material corto-punzantes en los trabajadores del área de emergencia en el Hospital León Becerra de la Ciudad de Milagro [Internet]. Guayaquil; 2013 [citado el 10 de marzo de 2015]. Disponible en: http://repositorio.unemi.edu.ec/ xmlui/handle/123456789/1268

15. Perú, Ministerio de Trabajo y Promoción del Empleo. Informe anual - El empleo en Perú y Lima Metropolitana - 2009 [Internet]. Lima: MINTRA; 2009 [citado el 10 de marzo de 2015]. Disponible en: http://www. trabajo.gob.pe/archivos/file/informes/ informe_anual_empleo_peru_lima_ metropolitana.pdf

16. Perú, Congreso de la República. Ley de Seguridad y Salud en el Trabajo, Ley 29783 [Internet]. Lima: 
El Peruano; 2011 [citado el 10 de marzo de 2015]. Disponible en: https://www.google.com.pe/ur1 ? sa $=t \& r c t=j \& q=\& e s r c=s \&$ sour $\mathrm{ce}=$ web $\& \mathrm{~cd}=1 \& \mathrm{cad}=$ rja $\&$ uact $=8 \&$ ved $=0 \mathrm{CB} s \mathrm{QFjAAahUKEwjb0PiO}$ se_HAhXGlA0KHXtnAZc\&url=http\%3A\%2F\%2Fwww.trabajo.gob. pe\%2Farchivos\%2Ffile\%2FSNIL\%2Fnormas\%2F2011-08-20_29783_1669. pdf\&usg=AFQjCNEtkhoCp_Uaf60usuo 4 x 17G7W3Pkw\&bvm = bv.102022582,bs.1,d.cWw

17. Rifkin J. El fin del trabajo. Nuevas tecnologías contra puestos de trabajo: el nacimiento de una nueva era. Revista Chilena de Derecho Informático. 2003;2:203-6.

18. España, Ministerio de Trabajo y Asuntos Sociales. Gestión de la prevención de riesgos laborales en la pequeña y mediana empresa. Madrid: MTAS; 2002.

19. Haro-García L, Aguilar-Madrid G, Juárez-Pérez CA, Aguilar-Rodríguez SD, Flores-Carbajal G, Gea-Izquierdo $\mathrm{E}$, et al. La equidad y la salud en el trabajo: explorando un área emergente. Rev Peru Med Exp Salud Publica. 2013;30(4):671-5.

20. Benavides FG, López-Ruiz M, Castejón E. Tendencia secular de las lesiones por accidentes de trabajo en España, 1904-2005. Arch Prev Riesgos Labor. 2008;11(3):141-7.

21. Velásquez A. La carga de enfermedad y lesiones en el Perú y las prioridades del plan esencial de aseguramiento universal. Rev Peru Med Exp Salud Publica. 2009;26(2):222-31.

22. García AM, Gadea R. Estimación de la mortalidad y morbilidad por enfermedades laborales en España. Arch Prev Riesgos Labor. 2004;7(1):38.

23. Contreras G, Torrealba B, Salinas M. Enfermedades respiratorias laborales en Chile: Ley 16.744. Rev Chil Enf Respir. 2014;30(1):27-34.

24. Díaz LM, Rendón LE. Un enfoque legal de los conceptos de accidente y enfermedad laboral en la jurisdicción legal colombiana [Internet]. Cali:
Universidad de San Buenaventura; 2014 [citado el 10 de marzo de 2015]. Disponible en: http:// bibliotecadigital.usbcali.edu.co:8080/ jspui/handle/10819/2440

25. Carrasco Cortez V, Lozano Salazar E, Velásquez Pancca E. Análisis actual y prospectivo de la oferta y demanda de médicos en el Perú 2005-2011. Acta Med Per. 2008;25(1):22-9.

26. García AM, Gadea R, López V. Estimación de la mortalidad atribuible a enfermedades laborales en España, 2004. Rev Esp Salud Pública. 2007;81(3):261-70.

Correspondencia: Christian R. Mejia.

Dirección: Av. Las Palmeras 5713 - Los Olivos. Lima 39 - Perú.

Teléfono: (511) 997643516

Correo electrónico: christian.mejia.md@ gmail.com

\section{Consulte la versión electrónica de la Revista Peruana de Medicina Experimental y Salud Pública en www.pubmed.gov}

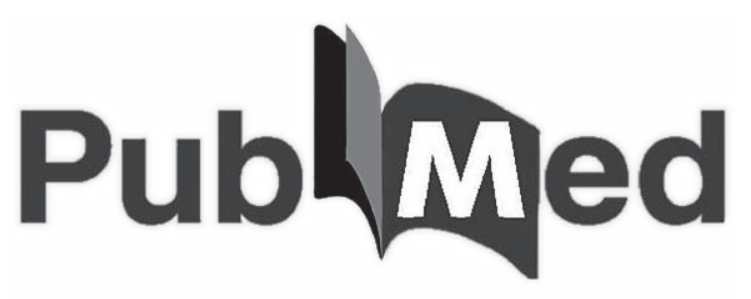

